\title{
Prognostic Significance of Transient Receptor Potential Vanilloid Type 1 (TRPV1) and Phosphatase and Tension Homolog (PTEN) in Epithelial Ovarian Cancer
}

\author{
GWAN HEE HAN ${ }^{1,2}$, DOO BYUNG CHAY ${ }^{1}$, SANGHEE NAM ${ }^{3}$, \\ HANBYOUL $\mathrm{CHO}^{1,4}$, JOON-YONG CHUNG ${ }^{4}$ and JAE-HOON KIM ${ }^{1}$ \\ ${ }^{1}$ Department of Obstetrics and Gynecology, Yonsei University College of Medicine, Seoul, Republic of Korea; \\ ${ }^{2}$ Department of Obstetrics and Gynecology, Kyung Hee University Hospital at Gangdong, Seoul, Republic of Korea; \\ ${ }^{3}$ Department of Obstetrics and Gynecology, Gangnam Severance Hospital, Seoul, Republic of Korea; \\ ${ }^{4}$ Experimental Pathology Laboratory, Laboratory of Pathology, Center for Cancer Research, \\ National Cancer Institute, National Institutes of Health, Bethesda, MD, U.S.A.
}

\begin{abstract}
Background: Transient receptor potential vanilloid type 1 (TRPV1) has been studied in human malignancies, but has not been studied in epithelial ovarian cancer (EOC). We, therefore, investigated the significance of TRPVI and correlation with phosphatase and tension homolog (PTEN) in EOC. Materials and Methods: Immunohistochemical analyses for TRPVI and PTEN were performed using a tissue microarray. Moreover, the role of TRPV1 in cell growth was assessed in a EOC cell line. Results: High TRPVI expression and the combination of high TRPVI and low PTEN expression were an independent prognostic factor for overall survival and disease-free survival. In vitro results demonstrated that knockdown of TRPVI was associated with decreased cell viability and colony formation. Conclusion: There is a strong association between TRPVI and PTEN and the combination of TRPVI and PTEN is a strong indicator of prognostic predictor in EOC.
\end{abstract}

Epithelial ovarian cancer (EOC) is the second most common gynecological malignancy in women, with a $1.3 \%$ prevalence, and one of the top leading causes of cancerrelated deaths in the United States of America (1). Women with EOC experience a high mortality rate because it is

This article is freely accessible online.

Correspondence to: Hanbyoul Cho, MD, Ph.D., Professor, Department of Obstetrics and Gynecology, Gangnam Severance Hospital, Yonsei University College of Medicine, 211 Eonjuro, Gangnam-Gu, Seoul 06273, Republic of Korea. Tel: +82 220193430, Fax: +82 234628209, email: hanbyoul@yuhs.ac

Key Words: Epithelial ovarian cancer, tumor marker, TRPV1, PTEN. usually diagnosed in advanced stages. Even with a greatly improved treatment strategy, the 5-year overall survival rate remains about $30 \%$ (2). Therefore, there is a great need for research studies that focus on uncovering the molecular pathogenesis of EOC and exploring more specific and effective prognostic markers to improve patient outcomes.

$\mathrm{Ca}^{2+}$ is one of the important second messengers linking activation of membrane receptor and downstream signaling cascade, and $\mathrm{Ca}^{2+}$ homeostasis controls various physiological and cellular processes such as tumorigenesis. Therefore, abnormal expression of $\mathrm{Ca}^{2+}$ channels has been studied in various cancers, and interest in the role of transient receptor potential (TRP) channels, a type of $\mathrm{Ca}^{2+}$ permeable nonselective cation channel, is also emerging. Transient receptor potential vanilloid type 1 (TRPV1), which belongs to the TRP channel family of proteins, was originally regarded as a critical sensor in response to mechanical, thermal, and chemical stimuli, since it is predominantly found in afferent neurons. It has been suggested that TRPV1 is associated with cell proliferation, differentiation, and invasion characteristics of cancer $(3,4)$. Previous studies have reported that TRPV1 acts as a tumor suppressor by inducing apoptosis and preventing tumor progression upon activation by its agonist in melanomas (5). However, other studies reported that TRPV1 deficiency prohibited cell proliferation in prostate cancer and sensitized stress-induced apoptosis in colorectal cancer, indicating an oncogenic role $(6,7)$. In accordance with previous studies, it can be suspected that the role of TRPV1 is tumor type-specific, and the mechanisms underlying the regulation of cancer cell growth by TRPV1 remain to be evaluated. Moreover, the role of TRPV1 in EOC has not been yet clarified.

Thus, in this study, we aimed to evaluate the molecular and functional significance of TRPV1 expression in EOC. Our results indicated that TRPV1 was overexpressed in EOC 
and associated with poor prognosis. Furthermore, in vitro functional studies showed that inhibition of TRPV1 suppressed the development of EOC cells.

\section{Materials and Methods}

Patients and tumor samples. Tissue samples from 217 EOCs, 57 borderline and 153 benign ovarian tumors, and 79 non-adjacent normal epithelia were included in the study. Tumor tissues were gathered from patients who underwent debulking surgery at the Gangnam Severance Hospital between 1996 and 2012 and from the Korea Gynecologic Cancer Bank as part of the Bio \& Medical Technology Development Program of the Ministry of the National Research Foundation (NRF) funded by the Korean government (MIST) (NRF-2017M3A9B8069610). The International Federation of Gynecology and Obstetrics (FIGO) classification was used for tumor staging. Clinical information, including survival time, survival status, age, and surgical procedure, were collected by reviewing medical records. The therapeutic response was evaluated with Response Evaluation Criteria in Solid Tumors (RECIST; version 1.0 ) by computed tomography (8). The cell type and tumor grade were obtained by reviewing the pathological reports. All tumor tissues were histologically examined by a gynecologic pathologist, and all biological samples were obtained after informed consent from the participants, according to the guidelines of the institutional review board (IRB) of Gangnam Severance Hospital.

Tissue microarray construction and immunohistochemistry. Tissue microarray (TMA) with one-millimeter cores was constructed from archival formalin-fixed paraffin-embedded (FFPE) tissue blocks. The samples were arrayed into a recipient paraffin block with a manual tissue arrayer MTA-1 (Beecher Instruments Inc., Silver Spring, MD, USA). For immunohistochemical staining, the TMA blocks were cut into serial $5-\mu \mathrm{m}$ thick sections, deparaffinized with xylene, and rehydrated gradually from ethanol to distilled water. Endogenous peroxidase activity was blocked in 3\% hydrogen peroxide $\left(\mathrm{H}_{2} \mathrm{O}_{2}\right)$ for $10 \mathrm{~min}$. The sections were immersed in an antigen retrieval buffer at $\mathrm{pH} 9$ (Dako, Carpinteria, CA, USA) and heated for $20 \mathrm{~min}$ in a steam pressure cooker (Pascal, Dako) to retrieve antigenicity. The sections were treated with a protein block (Dako) for 20 min to block non-specific staining. The sections were then incubated with anti-TRPV1 antibody (Alomone, Jerusalem, Israel; Rabbit polyclonal antibody, Cat\# ACC-030, 1:1,000 for $1 \mathrm{~h}$ ) and anti-PTEN antibody (Cell Signaling, Danvers, MA; Rabbit monoclonal antibody, Clone 9559L, 1:100 for $1 \mathrm{~h}$ ) in Dako Autostainer Plus (Dako) for $1 \mathrm{~h}$. For antigen-antibody reaction, sections were incubated with Dako EnVision+ Dual Link systemHRP (Dako) for 30 min and visualized with 3,3'-diaminobenzidine (DAB; Dako). Tissue sections were counterstained lightly with hematoxylin and examined with light microscopy. Appropriate negative controls were simultaneously prepared.

Evaluation of immunohistochemistry staining. After IHC staining, all glass slides were scanned digitally by a high-resolution optical scanner Nanozoomer 2.0 HT (Hamamatsu Photonics K.K., Japan). The digital imaging analysis (DIA) software used was the Visiopharm Integrator System v6.5.0.2303 (VIS; Visiopharm, Hørsholm, Denmark). After training the system with digital "painting" examples of the nucleus in the image, an algorithm for nucleus-specific signal selection was designed manually. The cytoplasm was defined by outlining the defined nucleus. TRPV1 and PTEN were scored based on staining intensity of brown-colored diaminobenzidine (DAB) ( 0 =negative, $1=$ weak, $2=$ moderate, and $3=$ strong). The overall immunostaining score was calculated by multiplying the staining intensity by percentage of positive cells (possible range, 0-300).

Cell culture. The human ovarian cancer cell line, A2780, was purchased from The European Collection of Cell cultures (ECACC, Salisbury, UK), and human embryonic kidney 293 (HEK293) cell from Systemic Biosciences (SBI, Palo Alto, CA, USA). A2780 cells were cultured at Roswell Park Memorial Institute (RPMI) in medium containing $10 \%$ FBS, $1 \%$ penicillin, and $1 \%$ streptomycin. The HEK293 cells were cultured in Dulbecco's modified Eagle's medium (DMEM) containing $1 \%$ penicillin and $1 \%$ streptomycin. A2780 cells and HEK293 cells were cultured at $37^{\circ} \mathrm{C}$ in a humidified atmosphere containing $5 \% \mathrm{CO}_{2}$.

Transfection and generation of stable cell lines. For the generation of sh-TRPV1 stable cell lines, pLKO.1 TRPVI shRNA libraries were purchased from Sigma-Aldrich (St. Louis, MO, USA). Of the lentivirus constructs tested, two with the best efficiency were used for the experiments here, using the human TRPVI sequence CCGGCCGT TTCATGTTTGTCTACATCTCGAGATGTAGACAAACATGAAACG GTTTT for shRNA\#1 and CCGGGAAGTTTATCTGCGACAG TTTCTCGAGAAACTGTCGCAGATAAACTTCTTTTTG for shRNA\#2. The non-targeted shRNA control vector (pLKO.1) was purchased from Sigma-Aldrich. Lentiviral production was done by cotransfecting pLKO.1 and packaging vectors into HEK293 cells by using Lipofectamine $^{\mathrm{TM}} 2000$ (Thermo Fisher Scientific, Waltham, MA, USA) according to the manufacturer's instructions. At 48 and $72 \mathrm{~h}$ posttransfection, virus particles were collected. A2780 cells were transduced, and cells with positive transduction were selected by puromycin $(2 \mu \mathrm{g} / \mathrm{ml})$.

Protein extraction and western blot. Total cell lysates were isolated by using cell lysis buffer [50 mM Tris $\mathrm{pH} 7.4,150 \mathrm{mM} \mathrm{NaCl}, 0.5 \%$ Triton X-100, $1 \mathrm{mM}$ ethylendiaminetetraacetic acid (EDTA)] containing proteinase inhibitor cocktail (Santa Cruz Biotechnology, Inc., Santa Cruz, CA, USA). The lysates were centrifuged at 13,500 rpm for $30 \mathrm{~min}$, and protein concentrations were determined by bicinchoninic acid (BCA) assay (Sigma-Aldrich). A total of $40 \mu \mathrm{g}$ proteins were separated by $8 \%$ sodium dodecyl sulfate polyacrylamide gel electrophoresis (SDS-PAGE) and transferred onto $0.2-\mu \mathrm{m}$ nitrocellulose membranes (Pall Corporation, Port Washington, NY, USA). The membranes were blocked with $5 \%$ nonfat dry milk in TBST mixture $(50 \mathrm{mM}$ Tris, $150 \mathrm{mM} \mathrm{NaCl}$, $0.1 \%$ Tween-20, $\mathrm{pH} 7.5$ ) for $1 \mathrm{~h}$ at room temperature, washed with TBST, and subsequently incubated with primary antibodies antiTRPV1 (Invitrogen, Carlsbad, CA, USA - Rabbit polyclonal antibody, Cat\# PA1-748) and $\alpha$-tubulin (Santa Cruz Biotechnology, Cat\# sc-5286). Primary antibodies against each protein were detected by using secondary antibodies conjugated with horseradish peroxidase. Signals were detected by chemiluminescence using the ImmunoCruz luminol reagent (Santa Cruz Biotechnology) and quantified using ImageJ analysis software (US National Institutes of Health, Bethesda, MD, USA).

Cell proliferation assay. Cell proliferation was measured with the EZ-CYTOX reagent (DoGenBio, Seoul, Republic of Korea, Cat\# EZ-3000). In brief, cells were seeded at $1 \times 10^{4}$ cells/well onto a 96 well plate with a final volume of $100 \mu \mathrm{l} /$ well. Cells were incubated 
at $37^{\circ} \mathrm{C}$ for $60 \mathrm{~min}$ after adding $10 \mu \mathrm{l}$ of EZ-CYTOX reagent per well and then incubated on an orbital shaker for $1 \mathrm{~min}$. The absorbance value at optical density (OD) $450 \mathrm{~nm}$ was subsequently measured with a microplate reader (Bio-Rad Laboratories, Inc., Hercules, CA, USA) and recorded at day 0, 1, 2, and 4. The experiment was performed in triplicates.

Colony formation assay. In order to examine clonogenicity, the cells were seeded in a 6 well plate at 500 cells/well. The cells were cultured in RPMI-1640 supplemented with $10 \%$ FBS and $1 \%$ penicillin/streptomycin for 2 weeks. The cells were fixed with $100 \%$ methanol for $10 \mathrm{~min}$ and stained with $0.5 \%$ crystal violet for 30 min, followed by washing with distilled water. Stained cells were dissolved in $2 \%$ dimethyl sulfoxide (DMSO) for $20 \mathrm{~min}$ on an orbital shaker. The absorbance value was measured at $595 \mathrm{~nm}$ on a microplate reader (Bio-Rad Laboratories, Inc.). Each experiment was repeated three times.

Cell counting assay. Cells were seeded at $2.5 \times 10^{5}$ cells/well in a 6well plate and incubated for 7 days. The cells were washed with phosphate-buffered saline and treated with trypsinethylenediaminetetraacetic acid. After detachment using trypsin, the cells were diluted with $1 \mathrm{ml}$ of fresh medium and transferred to the dedicated LUNA ${ }^{\mathrm{TM}}$ Cell Counting Slides (Logos Biosystems, Gyeonggi-do, Republic of Korea, Cat \#L12001). Cell counting was performed with the LUNA-II ${ }^{\mathrm{TM}}$ (Logos Biosystems) automated cell counter in disposable counting chambers. Each experiment was performed three times.

Statistical analysis. Statistical analyses of TRPV1 and PTEN were performed using the Mann-Whitney test or the KruskalWallis test where appropriate. Kaplan-Meier methods and logrank test were used to evaluate the overall survival (OS) and disease-free survival (DFS) curve. The Cox proportional hazard model was performed for both univariate and multivariate models to estimate hazard ratios (HRs) and confidence intervals (CIs). Statistical analyses were performed using SPSS version 25.0 (SPSS Inc., Chicago, IL, USA). A value of $p<0.05$ was considered statistically significant.

\section{Results}

TRPVI and PTEN protein expression in EOC. To investigate the clinical role of TRPV1 and PTEN in EOC, we assessed TRPV1 and PTEN expression by immunohistochemistry (IHC) using TMAs of 217 EOC tissues, 57 borderline tumors, 153 benign tumors, and 79 nonadjacent normal epithelial tissues. However, only 204 EOC tissues, 50 borderline tumors, 118 benign tumors, and 37 nonadjacent normal epithelial tissues for TRPV 1 and 202 EOC tissues, 50 borderline tumors, 95 benign tumors, and 79 nonadjacent normal epithelial tissues for PTEN were interpretable for IHC analysis due to complexity and staining of samples. Representative IHC images for TRPV1 and PTEN in EOC are shown in Figure 1A. As observed in Figure 1A and Table I, expression level of TRPV1 was higher compared to borderline tumors, benign tumors, and nonadjacent normal epithelial tissues (all $p<0.001$ ). We further evaluated the clinicopathological characteristics of TRPV1 in which high expression of TRPV1 (TRPV1+) was significantly associated with advanced FIGO stage ( $p<0.001$; Table I and Figure 1B), serous cell type $(p<0.001)$, and positive CA125 $(p=0.035$; Table I). In terms of PTEN, there were no significant differences in clinicopathological characteristics between EOC and other groups (Table I).

Correlation between TRPVI and PTEN expression. Since both TRPV1 and PTEN have been reported to be related to the PI3K/AKT pathway and $\mathrm{Ca}^{2+}$ homeostasis, Spearman's rank correlation analysis was performed to determine the association between the two proteins. The results showed a negative correlation trend between TRPV1 and PTEN in EOCs (Spearman's rho $=-0.103, p=0.144$; Figure 2A), but interestingly, significant negative correlation in stage III/IV was observed when performing subgroup analysis according to stage (Spearman's rho $=-0.241, p=0.006$; Figure 2B).

In addition, the combined high expression of TRPV1 and loss of PTEN expression (TRPV1+/PTEN-) was associated with advanced FIGO stage ( $p=0.013$; Figure $2 \mathrm{C}$ ) and serous cell type $(p=0.009$; Figure 2D). Other clinicopathological parameters were not found to be associated with TRPV1+/PTEN- expression in EOCs.

Prognostic significance of TRPVI and PTEN expression. We further evaluated the prognostic significance of TRPV1 and PTEN in EOCs, in which TPRV1+ patients had significantly worse OS compared to those with low TRPV1- $(p<0.001$; Figure 3A). In addition, DFS showed the same result as the OS $(p<0.001$; Figure 3B). Compared to TRPV1, high PTEN expression (PTEN+) showed significantly better DFS than low PTEN expression (PTEN-) $(p<0.001$; Figure 3D).

Moreover, OS and DFS were identified between TRPV1+/PTEN- and TRPV1-/PTEN+ expression groups with the Kaplan-Meier plot. Significant differences were observed in OS and DFS (both $p<0.001$; Figure $3 \mathrm{E}$ and Figure 3F). Furthermore, OS and DFS were compared with Cox proportional univariate and multivariate analyses (Table II). Multivariate analysis revealed that TRPV1+ expression strongly served as an independent prognostic factor for both OS and DFS (HR=4.19, 95\% CI $=1.34-12.18, p=0.009$; $\mathrm{HR}=8.22,95 \% \mathrm{CI}=3.37-20.04, p<0.001)$. The combined marker, TRPV1+/PTEN-, was related to poor DFS $(\mathrm{HR}=2.25,95 \% \mathrm{CI}=1.06-4.74, p=0.003)$ and in particular, TRPV+/PTEN - was a significantly strong prognostic biomarker for OS $(\mathrm{HR}=4.17,95 \% \mathrm{CI}=2.36-7.38, p<0.001)$. Moreover, FIGO stage and cell type were good parameters for predicting OS $(p=0.046, p<0.001)$, and other parameters, including FIGO stage $(p<0.001)$, cell type $(p=0.024)$ and tumor grade $(p=0.010)$, were independent poor prognostic factors for DFS. 

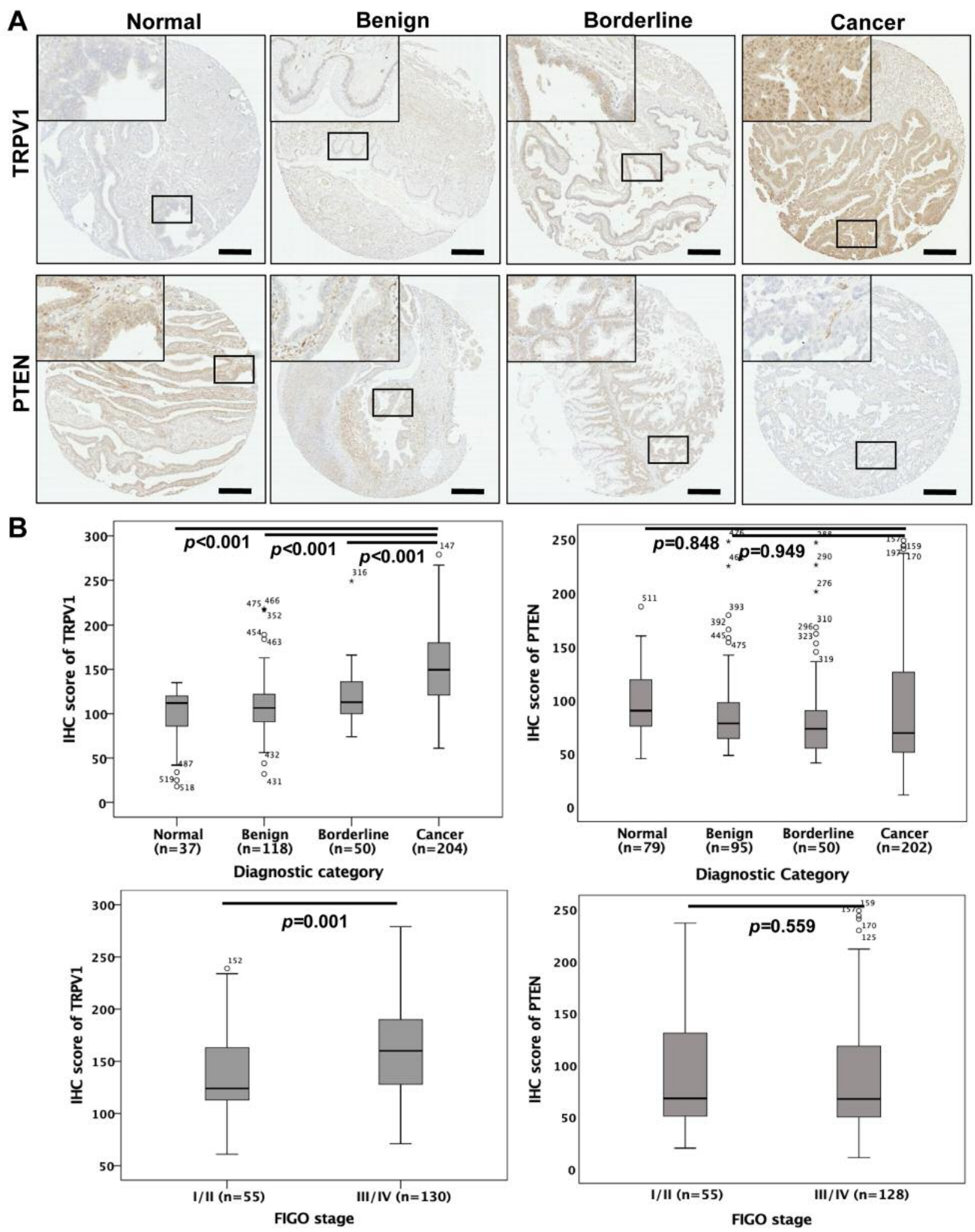

Figure 1. TRPVI and PTEN expression in formalin-embedded non-adjacent normal epithelium, benign tumor, borderline tumor, and epithelial ovarian cancer tissues. (A) Representative immunohistochemical images of TRPVI and PTEN in non-adjacent normal epithelium, benign tumor, borderline tumor, and epithelial ovarian cancer tissues. Scale bar: $60 \mu \mathrm{m}$. (B) IHC staining score for TRPV1 and PTEN in nonadjacent normal epithelium, benign tumor, borderline tumor, and epithelial ovarian cancer tissues. 
Table I. Expression of TRPVI and PTEN in relation to clinicopathological characteristics in immunohistochemical analysis.

\begin{tabular}{|c|c|c|c|c|c|c|}
\hline & \multirow[t]{2}{*}{ No. } & \multicolumn{2}{|c|}{ TRPV1 } & \multirow[t]{2}{*}{ No. } & \multicolumn{2}{|c|}{ PTEN } \\
\hline & & Mean score $(95 \% \mathrm{CI})$ & $p$-Value & & Mean score $(95 \% \mathrm{CI})$ & $p$-Value \\
\hline All study subjects & 409 & $132.1(127.9-136.3)$ & & 426 & $90.5(86.2-94.8)$ & \\
\hline \multicolumn{7}{|l|}{ Diagnostic category } \\
\hline Normal & 37 & $100.0(89.7-110.4)$ & $<0.001$ & 79 & $95.9(89.5-102.4)$ & 0.614 \\
\hline Benign & 118 & $109.3(103.6-115.0)$ & & 95 & $87.2(80.0-94.5)$ & \\
\hline Borderline & 50 & $118.3(110.5-126.1)$ & & 50 & $88.0(74.6-101.4)$ & \\
\hline Cancer & 204 & $154.5(148.6-160.4)$ & & 202 & $90.6(83.3-97.9)$ & \\
\hline FIGO stage & & & 0.001 & & & 0.559 \\
\hline I-II & 55 & $138.8(127.4-150.3)$ & & 55 & $92.2(77.5-107.2)$ & \\
\hline III-IV & 130 & $161.7(154.2-169.2)$ & & 128 & $87.2(78.2-96.3)$ & \\
\hline Cell type & & & $<0.001$ & & & 0.514 \\
\hline Serous & 137 & $166.6(159.6-173.5)$ & & 136 & $88.9(80.4-97.4)$ & \\
\hline Others & 67 & $129.8(121.3-138.4)$ & & 66 & $94.1(79.7-108.4)$ & \\
\hline Tumor grade & & & 0.673 & & & 0.612 \\
\hline Well/Moderate & 86 & $155.2(146.0-164.3)$ & & 86 & $88.1(77.0-99.3)$ & \\
\hline Poor & 102 & $157.8(149.4-166.2)$ & & 102 & $92.0(81.6-102.4)$ & \\
\hline CA125 & & & 0.035 & & & 0.636 \\
\hline Negative & 33 & $139.9(126.7-153.1)$ & & 33 & $93.8(76.8-110.8)$ & \\
\hline Positive & 167 & $157.2(150.5-163.9)$ & & 165 & $89.0(80.8-97.3)$ & \\
\hline Chemosensitivity & & & 0.710 & & & 0.536 \\
\hline Sensitive & 171 & $156.5(150.1-163.0)$ & & 170 & $92.2(84.0-100.4)$ & \\
\hline Resistant & 17 & $160.5(144.1-176.8)$ & & 16 & $83.5(57.8-109.3)$ & \\
\hline
\end{tabular}

FIGO, International Federation of Gynecology and Obstetrics; CI, confidence interval. Protein expression was evaluated by immunohistochemical analysis using tissue arrays, as described in Materials and Methods.

Knockdown of TRPV1 in EOC cells. As the biological role of TRPV1 in EOC is not fully investigated, we studied its potential roles in EOC cell lines by modulating intracellular TRPV1 expression with short- hairpin TRPV1 RNAi in a A2890 (sh-TRPV1 \#1, \#2) cell line. The relative expression level of TRPV1 was measured with western blot, and upon confirmation of knockdown (Figure 4A), we analyzed the effect of TRPV1 with two different assays to investigate the short- and long-term proliferative ability. When TRPV1 was knocked-down in A2890 cells, cell growth was significantly decreased compared to the shRNA-control group (Figure 4B). Next, we performed cell counting and colony formation assays to assess the role of TRPV1 in tumorigenesis and found that number of cells and colony formation in sh-TRPVI were significantly decreased compared to the shRNA-control (Figure 4C and D). Taken together, these results demonstrated that blocking TRPV1 expression inhibited cell growth in EOC cells.

\section{Discussion}

As EOC patients experience high rates of relapse and mortality, it is necessary to investigate the potential molecular biomarkers that can be of prognostic and predictive value for EOC. Therefore, in this study, we first identified the expression of TRPV1 and examined the impact on EOC progression and then studied the association of TRPV1 with PTEN and the potential combined effect of TRPV1 and PTEN as a prognostic marker for EOC.

First, we examined TRPV1 expression and explored its clinical significance with IHC analysis using EOCs, borderline tumors, benign tumors, and nonadjacent normal epithelia. The results revealed that TRPV1 expression was markedly increased in EOC tissues compared to borderline tumors, benign tumors, and nonadjacent normal epithelium (all $p<0.001$ ). Furthermore, TRPV1 expression increased in advanced FIGO stage and CA125-positive patients $(p<0.001$ and $p<0.035$, respectively). Notably, TRPV1+ status was an independent prognostic factor for both OS and DFS. In the same context, the expression of TRPV1 was studied in breast and prostate cancers and, as in our study, its expression was higher in cancer tissues than in normal tissues and was associated with poor prognosis (9-11). However, other studies on hepatocellular carcinoma, transitional cell carcinoma of bladder, and renal cell cancer have reported that expression of TRPV1 was inversely correlated with cancer progression (12-14). The main reason for this inconsistency would be that the hormones can modify transcription regulation, localization at the plasma membrane surface, and intrinsic activity of TRPV1 (15). Morelli et al. (6) evaluated androgen receptor (AR) and TRPV1 co- 

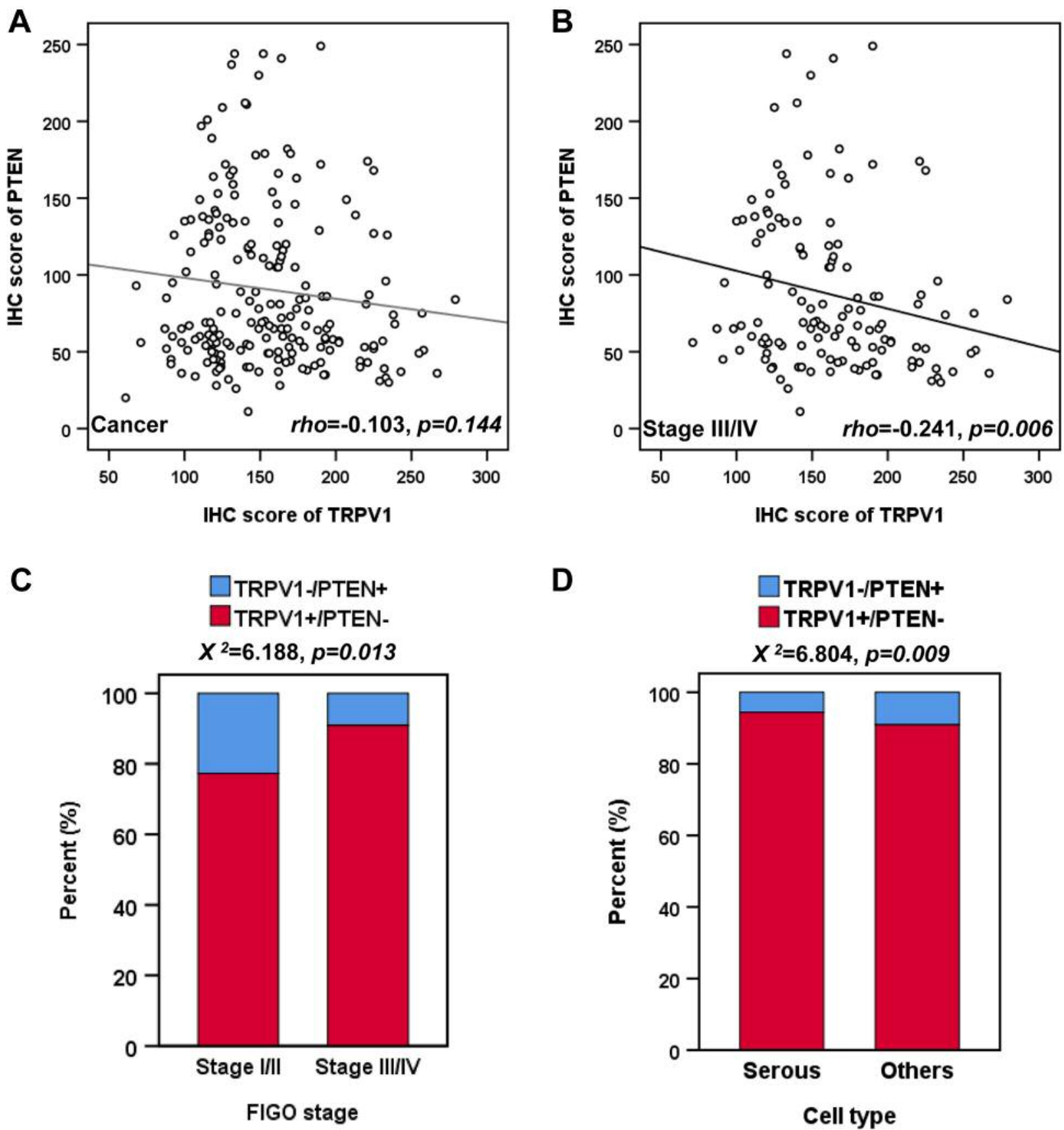

Figure 2. Spearman's rank correlation analysis between TRPV1 and PTEN expression. TRPV1 expression showed a trend of negative correlation with PTEN level in EOCs (A), while in stage III/IV, there was a significant negative correlation between TRPV1 and PTEN (B). TRPV1-/PTEN+ was significantly associated with FIGO stage $(C)$ and serous cell type $(D)$.

expression through mRNA and protein levels in advanced prostate cancer tissues, and found that expression levels of AR and TRPV1 were markedly elevated in these tissues compared to those in benign prostate hyperplasia. More importantly, they showed a strong correlation between AR and TRPV1 expression levels. Moreover, a strong down- regulation in AR and TRPV1 mRNA levels was observed in a patient who received androgen deprivation therapy compared to those who did not receive androgen deprivation therapy, suggesting that TRPV1 might be modulated by androgen. In this study, we did not show a correlation between TRPV1 and estrogen. The ovary, however, is a 
A

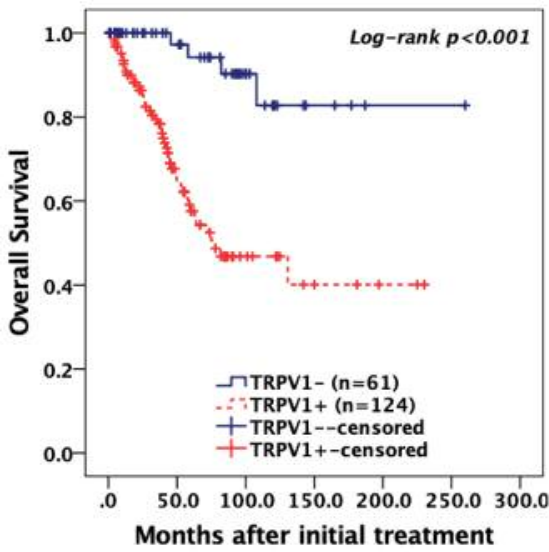

B

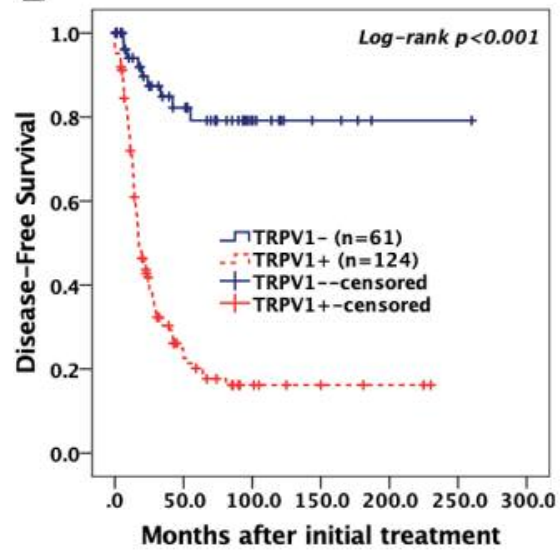

C

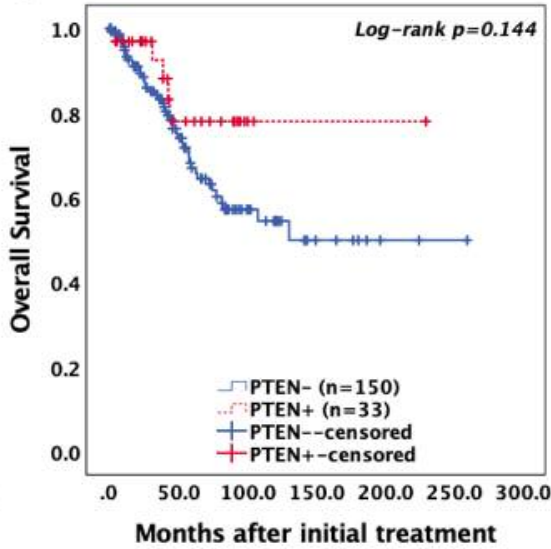

D

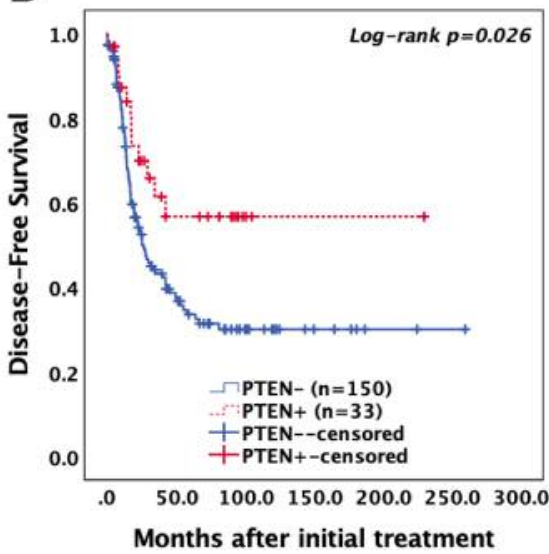

E

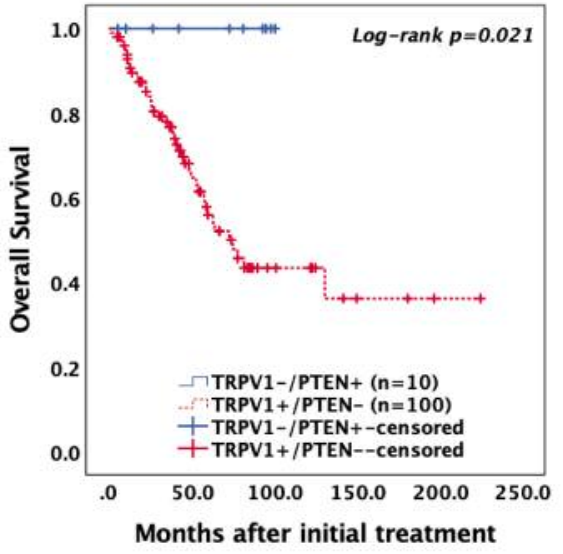

$\mathbf{F}$

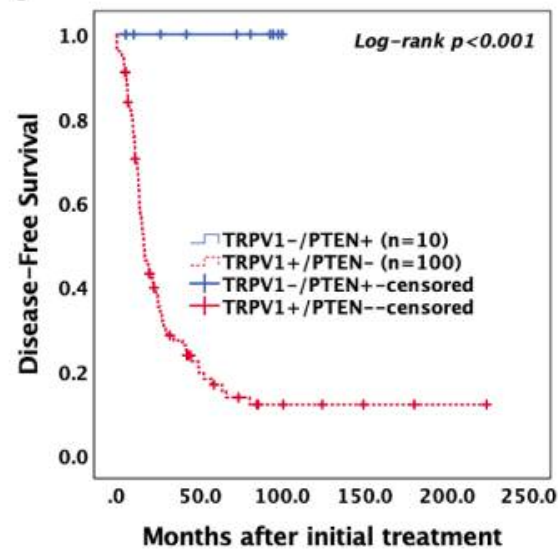

Figure 3. Kaplan-Meier survival curves for TRPV1 and PTEN expression in EOC. EOC patients with high TRPV1 expression had a shorter overall survival $(A)(p<0.001)$ and worse 5-year disease-free survival $(B)(p<0.001)$ than those with low TRPV1 expression. The patients with high expression of PTEN showed a longer 5-year disease-free survival $(D)(p=0.026)$ and those with high TRPV1/low PTEN had a shorter overall survival $(E)(p=0.021)$ and worse 5-year disease-free survival $(F)(p<0.001)$ than patients with low TRPV1/high PTEN expression.

Table II. Univariate and multivariate analyses of the associations between prognostic variables and overall and disease-free survival in epithelial ovarian cancer.

\begin{tabular}{lcccccc}
\hline & \multicolumn{2}{c}{ Overall survival hazard ratio $(95 \% \mathrm{CI}), p$-Value } & & \multicolumn{2}{c}{ Disease-free survival hazard ratio $(95 \% \mathrm{CI}), p$-Value } \\
\cline { 2 - 3 } & \multicolumn{2}{c}{ Univariate } & Multivariate & & Univariate & Multivariate \\
\hline FIGO stage (III-IV) & $4.68(1.85-11.79), 0.001$ & $2.66(1.01-6.99), 0.046$ & & $5.88(3.04-11.34),<0.001$ & $4.06(1.97-8.38),<0.001$ \\
Cell type (serous) & $4.71(1.87-11.87), 0.001$ & $2.01(0.75-5.40), 0.163$ & & $3.13(1.86-5.29),<0.001$ & $2.00(1.09-3.65), 0.024$ \\
Tumor grade (poor) & $1.69(0.95-3.01), 0.073$ & $\mathrm{NA}$ & & $1.96(1.29-2.99), 0.002$ & $1.76(1.14-2.70), 0.010$ \\
CA125+ $(>35 \mathrm{U} / \mathrm{ml})$ & $2.10(0.75-5.86), 0.153$ & $\mathrm{NA}$ & & $2.32(1.17-4.62), 0.016$ & $1.25(0.56-2.76), 0.582$ \\
Age $(>50)$ & $2.19(1.21-3.96), 0.010$ & $1.52(0.83-2.78), 0.166$ & & $1.55(1.04-2.31), 0.029$ & $1.29(0.84-1.98), 0.233$ \\
TRPV1+ & $7.18(2.57-20.01),<0.001$ & $4.18(1.43-12.18), 0.009$ & & $7.47(3.75-14.87),<0.001$ & $8.22(3.37-20.04),<0.001$ \\
PTEN_b & $1.96(0.77-4.95), 0.152$ & NA & & $1.96(1.07-3.58), 0.029$ & $1.83(0.96-3.48), 0.066$ \\
TRPV1+/PTEN- & $3.95(1.97-7.91),<0.001$ & $2.25(1.06-4.74), 0.033$ & & $4.42(2.74-7.12),<0.001$ & $4.17(2.36-7.38),<0.001$ \\
\hline
\end{tabular}

CI, Confidence interval; NA, not applicable; FIGO, International Federation of Gynecology and Obstetrics; LN, lymph node; NA, not applicable. acut-off value of TRPV1+ is 125 over IHC score; bcut-off of PTEN+ is 138 over IHC score. 
A
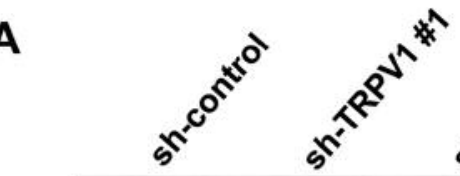

100

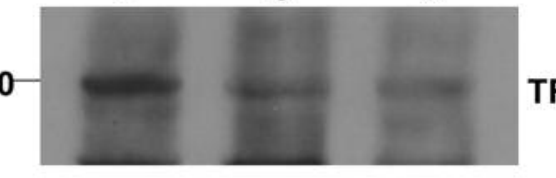

55

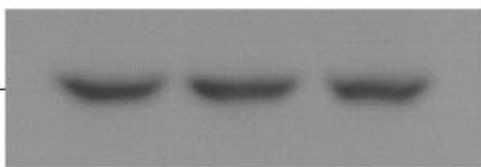

A2780

\section{B}

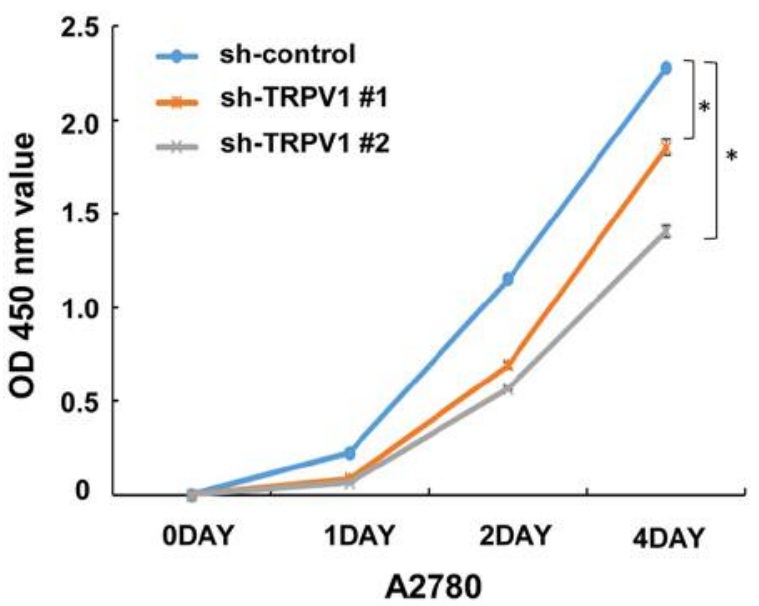

D

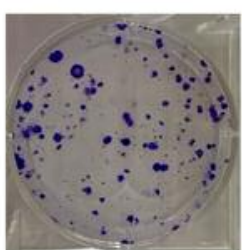

sh-control

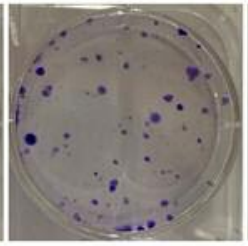

sh-TRPV1 \#1

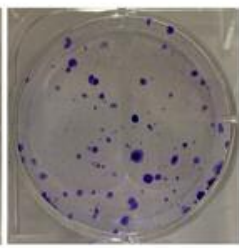

sh-TRPV1 \#2 a-tubulin

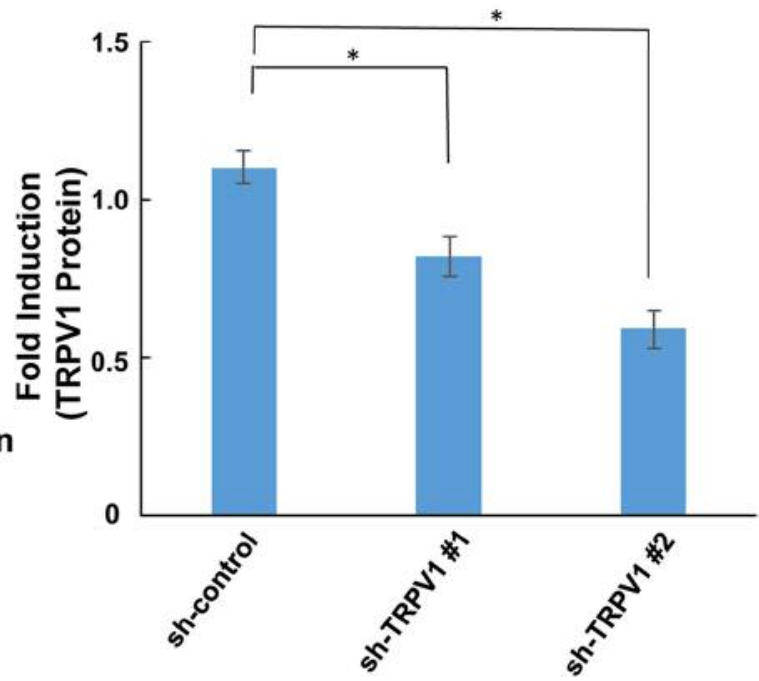

C

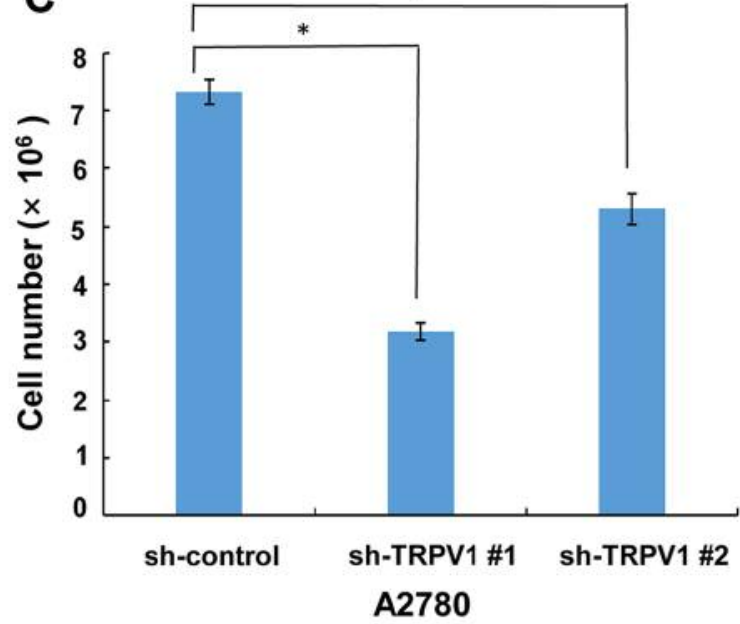

A2780

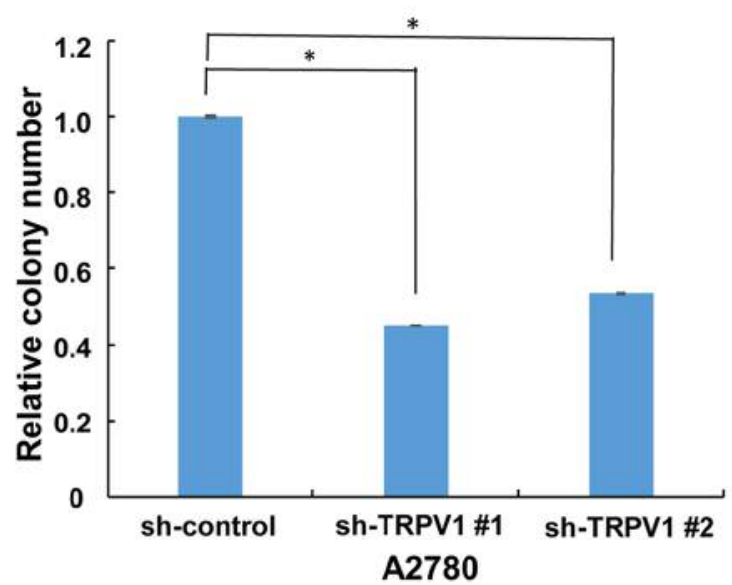

Figure 4. Effect of TRPV1 overexpression on EOC cells. Relative mRNA levels in control and TRPV1-knockdown A2780 cells (A). Viability curves for A2780 cells transfected with sh-control, sh-TRPV1 knockdown (B). A2780 cells were collected at various time points. The viability of TRPV1 knockdown cells was decreased. Quantification of cell counting assay for sh-control and sh-TRPV1-transfected A2980 cells (C). It showed significantly decreased cell number in sh-TRPV1 transfected A2980 cells compared to sh-control. The colony formation assay of sh-control and shTRPV1 transfected A2980 cells (D) Left panel: Representative images of colonogenic assay; Right panel: Quantitative results of colonogenic assay. Colony formation ability was decreased for the TRPV1 knockdown cells as compared with the control cells. The number of asterisks (*) indicates the level of significance: $* p \leq 0.05$. Data and error bars represent the mean \pm SD of triplicate experiments. 
hormone-affected reproductive organ, and synergetic expression of TRPV1 by estrogen has been reported in endometriosis patients (16). Therefore, accumulation of estrogen may have some influence on TRPV1 expression, and elevated expression of TRPV1 may promote expression of other $\mathrm{Ca}^{2+}$-sensitive transcriptional regulators $(17,18)$.

Another important finding of this study is that expression of TRPV1 was significantly higher in serous-type EOC than in others. The fourth edition of WHO classification placed ovarian cancer into two different categories, type I and type II, according to molecular profile, precursor lesion, way of spread, and response to chemotherapy (19). Type 1 EOC includes low-grade serous ovarian carcinoma (LGOSC), mucinous, clear cell, malignant Brenner and endometrioid tumors that generally show Ras and/or Raf mutation with indolent and slow growth. Type II EOC, which exhibits p53 mutations, includes high-grade serous ovarian carcinoma (HGOSC) undifferentiated carcinomas, and malignant mixed mesodermal tumors. Among the many types of EOC, HGOSC accounts for nearly $90 \%$ and exhibits a more aggressive behavior. Despite continued research, there has been no significant improvement in 5-year OS over the past 30 years. Therefore, it is necessary to identify a potential biomarker that correlates with prognosis in Type II EOC, especially HGOSC, in the future (20). In our study, among 137 serous type EOC patients, $13.7 \%$ had LGOSC and $85.1 \%$ had HGOSC (1.2\%: grade unknown). Taken together, our results suggest that TRPV1 might be a crucial predictor for poor prognosis of HGOSC and possible therapeutic target.

In accordance with our results showing an association between TRPV1 and cancer in clinical specimens, we set out to explore the potential role of TRPV1 in the development of a malignant phenotype in EOC cells by modulating intracellular TRPV1 expression in A2890 cells. We found that TRPV1 knockdown significantly decreased the proliferative and clonogenic abilities of EOC cells. Similar to our results, accumulative evidence has demonstrated the role of TRPV1 in tumorigenesis of various cancers. Sung et al. (7) identified the oncogenic role of TRPV1 in colorectal cancer after observing that tumor necrosis factor-related apoptosis-inducing ligand (TRAIL) was activated and induced cell apoptosis when TRPV1 expression was blocked by an antagonist in colorectal cells. Moreover, in prostate cancer cells, TRPV1 overexpression activated its oncogenic role via regulated extracellular signal-regulated kinase (ERK) signaling (6). However, in glioma, TRPV1 overexpression leads to cell death by inducing endoplasmic reticulum stress (3). de Jong et al. (21) found that activation of TRPV1 by capsaicin regulated $\mathrm{Ca}^{2+} /$ calpain and protein tyrosine phosphatase 1B (PTP1B) and suppressed intestinal tumorigenesis. Considering these inconsistent results from previous studies, we suspect that TRPV1 can show varying functions depending on cancer type. One factor that could affect TRPV1 function is tumor microenvironment, since cytokine and receptor interactions vary by tumor type and tissue content and consequently affect the different cancerrelated pathways in tumor microenvironments (22).

In addition to TRPV1, we investigated the expression of PTEN and its association with TRPV1 in EOC. A dual lipidprotein phosphatase, PTEN acts primarily as a tumor suppressor by dysregulating the $\mathrm{PI} 3 \mathrm{~K} / \mathrm{AKT} / \mathrm{mTOR}$ pathway in a large proportion of human cancers such as prostate, brain, breast, thyroid, endometrial, sarcoma, and cervical cancers (23-26). In our study, the expression levels of PTEN did not differ between normal and cancer tissues. However, Cox analysis revealed that low expression of PTEN is an independent predictive factor for prognosis. These results may be explained by the fact that more than $70 \%$ of PTEN alterations are related to gene deletions that were determined to yield intact PTEN by IHC, and only $30 \%$ are related to lack of PTEN expression according to the TCGA database (27). Moreover, for HGOSC, PTEN IHC in TCGA data showed loss of PTEN in only 52 (15\%) out of the 316 EOCs (28).

After looking at the expression and survival analyses of TRPV1 and PTEN, we examined the relationship between TRPV1 and PTEN in EOCs, which showed a significant negative correlation in advanced stages (stage III/IV). In addition, combination of TRPV1 and PTEN (TRPV1+/PTEN) showed high hazard ratio in the Cox regression analyses, indicating a strong prognostic predictive factor. As mentioned above, the association between TRPV1 and PTEN can be observed through the PI3K/AKT pathway, since PTEN acts as a tumor suppressor by inhibiting cell proliferation and induces apoptosis via dephosphorylation of phosphatidylinositol $(3,4,5)$-triphosphates to phosphatidylinositol 4,5-bisphosphate in the PI3K/AKT pathway. A previous study also revealed that activation of TRPV1 may exert anti-apoptotic effects in ischemic/reperfusion injury of the heart via the PI3K/AKT pathway (29). In addition to this result, numerous studies have reported on the association of TRPV1 and the PI3K/AKT pathway such as in dorsal root ganglion neurons (30) and human HepG2 cells $(30,31)$. Taken together, we propose that TRPV1 and PTEN showed correlation in our EOC specimens through the PI3K/AKT pathway. Even though there is no previous study investigating the correlation between TRPV1 and PTEN expression in various cancers, the study by Liu et al. (32) is of particular interest. They observed that overexpression of TRPV4 in colon cancer was associated with poor prognosis and revealed the negative regulation of PTEN upon TRPV4 activation via the AKT/mammalian target of rapamycin (mTOR) signaling pathway. Furthermore, they suggested that dysregulation of $\mathrm{Ca}^{2+}$ homeostasis by TRPV4 regulated the localization of PTEN by conformational changes in major vault proteins in colon cancer. However, further studies are warranted to understand the exact mechanism underlying the role of TRPV1 and PTEN in EOC. 
In this study, the evaluation of TRPV1 and PTEN expression level in EOCs were performed by IHC. Immunostaining techniques, IHC is the most practical methods to assess expression levels of proteins in histopathology. It is relatively inexpensive and easy to perform, and IHC provides not only a semiquantitative assessment but also defines cellular localization of protein expression. However, the determined TRPV1 and PTEN expression accurately by IHC can be significantly affected by numerous factors, such as temperature, fixation and method of antigen retrieval $(33,34)$. Therefore, to reduce the variability in scoring result of $\mathrm{IHC}$, recommendation guidelines should be published.

In conclusion, we investigated TRPV1 and PTEN expression using IHC in a large cohort of patients with EOC. High TRPV1 expression was associated with poor DFS and OS, and high PTEN expression showed a favorable prognosis in DFS. Cox regression analysis confirmed that both TRPV1 and PTEN might be important prognostic indicators of EOC. This result is significant because it suggests that TRPV1 or combination of TRPV1 and PTEN is applicable to the personalized therapy approach based on the constancy of tumors.

\section{Conflicts of Interest}

The Authors do not have any potential conflicts of interest to disclose.

\section{Authors' Contributions}

$\mathrm{HC}$ and J-YC designed and built tissue microarrays. GHH, HC, and $\mathrm{J}-\mathrm{YC}$ conceived and designed the study, and modified the experimental design. GHH, and DC performed data analysis for experiments or clinical records. SN performed functional studies. GHH and DC drafted the manuscript and figure legend. J-HK, J-YC, and HC revised the figures and added critical contents to the discussion, and were responsible for revising all portions of the submitted manuscript. All Authors read and approved the final manuscript.

\section{Acknowledgements}

This research was supported by the Bio \& Medical Technology Development Program of the National Research Foundation (NRF) funded by the Korean government (MSIT) (NRF-2017M3A9B8 069610). This study was also supported by a faculty research grant of Yonsei University College of Medicine (6-2017-0160).

\section{References}

1 Jemal A, Siegel R, Xu J and Ward E: Cancer statistics, 2010. CA Cancer J Clin 60(5): 277-300, 2010. PMID: 20610543. DOI: $10.3322 /$ caac. 20073

2 Eisenhauer EA: Real-world evidence in the treatment of ovarian cancer. Ann Oncol 28(suppl_8): viii61-viii65, 2017. PMID: 29232466. DOI: 10.1093/annonc/mdx443

3 Amantini C, Mosca M, Nabissi M, Lucciarini R, Caprodossi S, Arcella A, Giangaspero F and Santoni G: Capsaicin-induced apoptosis of glioma cells is mediated by TRPV1 vanilloid receptor and requires p38 MAPK activation. J Neurochem 102(3): 977-990, 2007. PMID: 17442041. DOI: 10.1111/j.14714159.2007.04582.x

4 Berridge MJ, Bootman MD and Roderick HL: Calcium signalling: dynamics, homeostasis and remodelling. Nat Rev Mol Cell Biol 4(7): 517-529, 2003. PMID: 12838335. DOI: 10.1038/nrm1155

5 Yang Y, Guo W, Ma J, Xu P, Zhang W, Guo S, Liu L, Ma J, Shi Q, Jian Z, Liu L, Wang G, Gao T, Han Z and Li C: Downregulated TRPV1 Expression Contributes to Melanoma Growth via the Calcineurin-ATF3-p53 Pathway. J Invest Dermatol 138(10): 2205-2215, 2018. PMID: 29580868. DOI: 10.1016/j.jid.2018.03.1510

6 Morelli MB, Amantini C, Nabissi M, Liberati S, Cardinali C, Farfariello V, Tomassoni D, Quaglia W, Piergentili A, Bonifazi A, Del Bello F, Santoni M, Mammana G, Servi L, Filosa A, Gismondi A and Santoni G: Cross-talk between alpha1Dadrenoceptors and transient receptor potential vanilloid type 1 triggers prostate cancer cell proliferation. BMC Cancer 14: 921, 2014. PMID: 25481381. DOI: 10.1186/1471-2407-14-921

7 Sung B, Prasad S, Ravindran J, Yadav VR and Aggarwal BB: Capsazepine, a TRPV1 antagonist, sensitizes colorectal cancer cells to apoptosis by TRAIL through ROS-JNK-CHOPmediated upregulation of death receptors. Free Radic Biol Med 53(10): 1977-1987, 2012. PMID: 22922338. DOI: 10.1016/ j.freeradbiomed.2012.08.012

8 Therasse P, Arbuck SG, Eisenhauer EA, Wanders J, Kaplan RS, Rubinstein L, Verweij J, Van Glabbeke M, van Oosterom AT, Christian MC and Gwyther SG: New guidelines to evaluate the response to treatment in solid tumors. European Organization for Research and Treatment of Cancer, National Cancer Institute of the United States, National Cancer Institute of Canada. J Natl Cancer Inst 92(3): 205-216, 2000. PMID: 10655437. DOI: 10.1093/jnci/92.3.205

9 Weber LV, Al-Refae K, Wolk G, Bonatz G, Altmuller J, Becker C, Gisselmann $G$ and Hatt $\mathrm{H}$ : Expression and functionality of TRPV1 in breast cancer cells. Breast Cancer (Dove Med Press) 8: 243-252, 2016. PMID: 28008282. DOI: 10.2147/BCTT.S 121610

10 Czifra G, Varga A, Nyeste K, Marincsak R, Toth BI, Kovacs I, Kovacs L and Biro T: Increased expressions of cannabinoid receptor-1 and transient receptor potential vanilloid-1 in human prostate carcinoma. J Cancer Res Clin Oncol 135(4): 507-514, 2009. PMID: 18830626. DOI: 10.1007/s00432-008-0482-3

11 Clark R and Lee SH: Anticancer properties of capsaicin against human cancer. Anticancer Res 36(3): 837-843, 2016. PMID: 26976969.

12 Miao X, Liu G, Xu X, Xie C, Sun F, Yang Y, Zhang T, Hua S, Fan W, Li Q, Huang S, Wang Q, Liu G and Zhong D: High expression of vanilloid receptor-1 is associated with better prognosis of patients with hepatocellular carcinoma. Cancer Genet Cytogenet 186(1): 25-32, 2008. PMID: 18786439. DOI: 10.1016/j.cancergencyto.2008.05.011

13 Lazzeri M, Vannucchi MG, Spinelli M, Bizzoco E, Beneforti P, Turini D and Faussone-Pellegrini MS: Transient receptor potential vanilloid type 1 (TRPV1) expression changes from normal urothelium to transitional cell carcinoma of human bladder. Eur Urol 48(4): 691-698, 2005. PMID: 15992990. DOI: 10.1016/j.eururo.2005.05.018 
14 Wu YY, Liu XY, Zhuo DX, Huang HB, Zhang FB and Liao SF: Decreased expression of TRPV1 in renal cell carcinoma: association with tumor Fuhrman grades and histopathological subtypes. Cancer Manag Res 10: 1647-1655, 2018. PMID: 29970964. DOI: $10.2147 / C M A R . S 166390$

15 Gkika D and Prevarskaya N: Molecular mechanisms of TRP regulation in tumor growth and metastasis. Biochim Biophys Acta 1793(6): 953-958, 2009. PMID: 19103233. DOI: 10.1016/j.bbamcr.2008.11.010

16 Greaves E, Grieve K, Horne AW and Saunders PT: Elevated peritoneal expression and estrogen regulation of nociceptive ion channels in endometriosis. J Clin Endocrinol Metab 99(9): E17381743, 2014. PMID: 25029427. DOI: 10.1210/jc.2014-2282

17 Remoue F, Jacobs N, Miot V, Boniver J and Delvenne P: High intraepithelial expression of estrogen and progesterone receptors in the transformation zone of the uterine cervix. Am J Obstet Gynecol 189(6): 1660-1665, 2003. PMID: 14710094.

18 Park YR, Chun JN, So I, Kim HJ, Baek S, Jeon JH and Shin SY: Data-driven analysis of TRP channels in cancer: Linking variation in gene expression to clinical significance. Cancer Genomics Proteomics 13(1): 83-90, 2016. PMID: 26708603.

19 Berns EM and Bowtell DD: The changing view of high-grade serous ovarian cancer. Cancer Res 72(11): 2701-2704, 2012. PMID: 22593197. DOI: 10.1158/0008-5472.CAN-11-3911

20 Ahmed AA, Etemadmoghadam D, Temple J, Lynch AG, Riad M, Sharma R, Stewart C, Fereday S, Caldas C, Defazio A, Bowtell $\mathrm{D}$ and Brenton JD: Driver mutations in TP53 are ubiquitous in high grade serous carcinoma of the ovary. J Pathol 221(1): 4956, 2010. PMID: 20229506. DOI: 10.1002/path.2696

21 de Jong PR, Takahashi N, Harris AR, Lee J, Bertin S, Jeffries J, Jung M, Duong J, Triano AI, Lee J, Niv Y, Herdman DS, Taniguchi K, Kim CW, Dong H, Eckmann L, Stanford SM, Bottini N, Corr M and Raz E: Ion channel TRPV1-dependent activation of PTP1B suppresses EGFR-associated intestinal tumorigenesis. J Clin Invest 124(9): 3793-3806, 2014. PMID: 25083990. DOI: $10.1172 /$ JCI72340

22 Bertin S, Aoki-Nonaka Y, de Jong PR, Nohara LL, Xu H, Stanwood SR, Srikanth S, Lee J, To K, Abramson L, Yu T, Han T, Touma R, Li X, Gonzalez-Navajas JM, Herdman S, Corr M, Fu G, Dong H, Gwack Y, Franco A, Jefferies WA and Raz E: The ion channel TRPV1 regulates the activation and proinflammatory properties of CD4(+) T cells. Nat Immunol 15(11): 1055-1063, 2014. PMID: 25282159. DOI: $10.1038 /$ ni.3009

23 Akca H, Demiray A, Aslan M, Acikbas I and Tokgun O: Tumour suppressor PTEN enhanced enzyme activity of GPx, SOD and catalase by suppression of PI3K/AKT pathway in non-small cell lung cancer cell lines. J Enzyme Inhib Med Chem 28(3): 539-544, 2013. PMID: 22299584. DOI: 10.3109/14756366.2011.654114

$24 \mathrm{Bu} \mathrm{W}$ and Luo T: miR-1297 promotes cell proliferation of nonsmall cell lung cancer cells: Involving in PTEN/Akt/Skp2 signaling pathway. DNA Cell Biol 36(11): 976-982, 2017. PMID: 28872922. DOI: 10.1089/dna.2017.3886.

25 Kechagioglou P, Papi RM, Provatopoulou X, Kalogera E, Papadimitriou E, Grigoropoulos P, Nonni A, Zografos G, Kyriakidis DA and Gounaris A: Tumor suppressor PTEN in breast cancer: heterozygosity, mutations and protein expression. Anticancer Res 34(3): 1387-1400, 2014. PMID: 24596386.
26 Lim HJ, Wang X, Crowe P, Goldstein D and Yang JL: Targeting the PI3K/PTEN/AKT/mTOR Pathway in Treatment of Sarcoma Cell Lines. Anticancer Res 36(11): 5765-5771, 2016. PMID: 27793898. DOI: 10.21873 /anticanres. 11160

27 Cancer Genome Atlas Research N: Integrated genomic analyses of ovarian carcinoma. Nature 474(7353): 609-615, 2011. PMID: 21720365. DOI: $10.1038 /$ nature 10166

28 Hanrahan AJ, Schultz N, Westfal ML, Sakr RA, Giri DD, Scarperi S, Janakiraman M, Olvera N, Stevens EV, She QB, Aghajanian C, King TA, Stanchina E, Spriggs DR, Heguy A, Taylor BS, Sander C, Rosen N, Levine DA and Solit DB: Genomic complexity and AKT dependence in serous ovarian cancer. Cancer Discov 2(1): 56-67, 2012. PMID: 22328975. DOI: 10.1158/2159-8290.CD-11-0170

29 Jiang XX, Liu GY, Lei H, Li ZL, Feng QP and Huang W: Activation of transient receptor potential vanilloid 1 protects the heart against apoptosis in ischemia/reperfusion injury through upregulating the PI3K/Akt signaling pathway. Int J Mol Med 41(3): 1724-1730, 2018. PMID: 29286076. DOI: 10.3892/ijmm.2017.3338

30 Tang HB and Nakata Y: The activation of transient receptor potential vanilloid receptor subtype 1 by capsaicin without extracellular $\mathrm{Ca} 2+$ is involved in the mechanism of distinct substance $\mathrm{P}$ release in cultured rat dorsal root ganglion neurons. Naunyn Schmiedebergs Arch Pharmacol 377(4-6): 325-332, 2008. PMID: 18034335. DOI: 10.1007/s00210-007-0211-5

31 Joung EJ, Li MH, Lee HG, Somparn N, Jung YS, Na HK, Kim $\mathrm{SH}$, Cha YN and Surh YJ: Capsaicin induces heme oxygenase1 expression in HepG2 cells via activation of PI3K-Nrf2 signaling: NAD(P)H:quinone oxidoreductase as a potential target. Antioxid Redox Signal 9(12): 2087-2098, 2007. PMID: 17979524. DOI: 10.1089/ars.2007.1827

32 Liu X, Zhang P, Xie C, Sham KWY, Ng SSM, Chen Y and Cheng CHK: Activation of PTEN by inhibition of TRPV4 suppresses colon cancer development. Cell Death Dis 10(6): 460, 2019. PMID: 31189890. DOI: 10.1038/s41419-019-1700-4

33 Ross JS and Fletcher JA: HER-2/neu (c-erb-B2) gene and protein in breast cancer. Am J Clin Pathol 112(1 Suppl 1): S5367, 1999. PMID: 10396301.

34 Rattan B, Manjari M, Kahlon SK, Kalra N, Bhalla A and Paul S: The Immunohistochemical Expression of the Oestrogen Receptor (ER), HER-2/NEU and Cytokeratin 8/18 and 5/6 in Invasive Breast Carcinoma. J Clin Diagn Res 6(9): 1495-1498, 2012. PMID: 23285439. DOI: $10.7860 / J C D R / 2012 / 4086.2542$

Received February 9, 2020

Revised February 22, 2020

Accepted March 6, 2020 